Relations industrielles

Industrial Relations

\title{
Helly, Denise, et Alberte Ledoyen, Immigrés et créations d'entreprises : Montréal 1990
}

\section{Zhan Su}

Volume 50, numéro 3, 1995

URI : https://id.erudit.org/iderudit/051046ar

DOI : https://doi.org/10.7202/051046ar

Aller au sommaire du numéro

Éditeur(s)

Département des relations industrielles de l'Université Laval

ISSN

0034-379X (imprimé)

1703-8138 (numérique)

Découvrir la revue

Citer ce compte rendu

Su, Z. (1995). Compte rendu de [Helly, Denise, et Alberte Ledoyen, Immigrés et créations d'entreprises : Montréal 1990]. Relations industrielles / Industrial Relations, 50(3), 672-675. https://doi.org/10.7202/051046ar

Tous droits réservés @ Département des relations industrielles de l'Université Laval, 1995
Ce document est protégé par la loi sur le droit d'auteur. L’utilisation des services d'Érudit (y compris la reproduction) est assujettie à sa politique d'utilisation que vous pouvez consulter en ligne.

https://apropos.erudit.org/fr/usagers/politique-dutilisation/ 
d'intervention (M. Laflamme, "La qualité totale: un nouveau paradigme pour les organisations ", Revue Organisation, automne 1994, p. 13-25).

À notre avis, l'auteur n'a pas su bien cerner la spécificité de la qualité totale ; il l'associe à la reprise de thèmes traditionnels (p. 19) et fait une mauvaise distinction entre les concepts de productivité et de qualité (p.16). Nous concluons que l'auteur n'a pas atteint les objectifs (1) et (3) de son étude (p. 29), soit: "Préciser tout le débat actuel sur la qualité des services publics à l'aide des idées et des critiques formulées par certains auteurs et, d'autre part, des modèles théoriques et des concepts qui se dégagent le mieux de tous les écrits. [...] Dégager un ou des modèles généraux pour servir de guides à d'autres organisations qui veulent tendre à un même but. "

Cependant, le second objectif de volume a bien été complété, soit : "à partir de cas réels, décrire et analyser les expériences vécues par certaines organisations publiques qui se sont lancées dans un tel projet". Sur ce point, nous félicitons l'auteur, qui fait oeuvre de pionnier dans ce domaine au Québec et nous souhaitons par cette occasion la publication d'autres ouvrages qui permettent de mieux maîtriser ce phénomène si complexe de la qualité totale.
$\grave{A}$ cet effet, je termine par deux suggestions:

1) Pour les auteurs : retourner aux sources, constituer des équipes multidisciplinaires et aller voir ce qui se passe dans le secteur privé où le mouvement qualité a débuté et a pris une longueur d'avance sur le public (exemples : Xérox et Venmar).

2) Pour les organismes publics : si l'on veut prendre la qualité totale au sérieux, il faut effectuer dans une première phase un diagnostic des coûts cachés de la non-qualité (environ $15 \%$ à $40 \%$ des frais d'exploitation) qui seraient évalués par une firme indépendante (externe). Par la suite, pourrait suivre la démarche d'amélioration continue pour réduire ces coûts cachés à moins de $10 \%$ du coût d'exploitation. Actuellement les consultants en qualité sont embauchés par les gestionnaires-fonctionnaires qui ne prennent naturellement que ce qui leur va dans la panoplie des programmes en qualité totale. C'est pourquoi il est si imprudent de juger le mouvement de la qualité totale à partir d'expériences de courtes durées et implantées de façon fragmentaire.

MARCEL LAFLAMME

Université de Sherbrooke

\section{Immigrés et création d'entreprises: Montréal 1990}

par Denise HELLY et Alberte LEDOYEN, Québec, IQRC, 1994, 305 p., ISBN 289224-191-X.

Le livre que nous proposent Helly et Ledoyen est basé sur un projet de recherche subventionné par la Direction de la recherche du ministère des Communautés culturelles et de l'Immigration du Québec. Il a pour objet d'approfondir les quelques points importants souvent abordés dans la littérature sur l'entrepreneuriat immigré, à savoir les raisons qui poussent les immigrés à entreprendre, le choix du sous-secteur d'activités et l'or- ganisation de la mobilisation des ressources utiles à la création d'une entreprise. Précisons que cette recherche porte exclusivement sur les immigrés entrepreneurs qui s'installent à Montréal, et qu'elle tient davantage compte du nouveau contexte canadien à la suite des changements de conjoncture, voire de structure, économique survenus durant les années 1970 . 
Les quatorze chapitres de ce livre sont divisés en trois parties. Dans la première partie, les auteurs débutent avec une explication des objectifs et des hypothèses de leur recherche formulées à partir de la littérature existante. Ensuite, elles présentent le profil de la population étudiée. Cette dernière est composée d'un échantillon de 203 individus immigrants minutieusement sélectionnés : ils ont créé à Montréal leur premier emploi autonome ou leur première entreprise entre 1982 et 1986 , et ce, de façon viable; ils travaillent dans des sous-secteurs très variés, aussi bien dans ceux requérant du capital ou de l'expertise que dans ceux dont les barrières à l'entrée sont très faibles; ils appartiennent soit à des groupes au sein desquels une tradition d'entrepreneuriat existait durant les années de leur arrivée à Montréal, soit à ceux au sein desquels cette tradition n'existait pas; ils ont été formés soit à l'étranger avant l'immigration, soit au Québec après l'immigration. Voilà donc déjà une particularité intéressante de cette recherche, sachant que, jusqu'à maintenant, les rares études disponibles en la matière se limitent très souvent à des analyses générales de type macro, ou bien à un petit nombre de sous-secteurs ou de personnes peu significatifs.

La deuxième partie du livre traite essentiellement des raisons de la création d'un premier emploi autonome ou d'une première entreprise par des immigrés. Pour ce faire, les auteures se livrent d'abord à une analyse de corrélation entre, d'une part, les types d'itinéraires occupationnels suivis par les immigrés avant la création de leur entreprise et d'autre part, la nature de leurs ressources à l'arrivée (capital, métier ou expertise) et la présence ou non de désavantages liés à l'immigration (handicaps personnels : charge familiale et âge avancé ; obstacles structurels : méconnaissance de la langue officielle et nonreconnaissance de l'expertise). Un exercice intéressant, mais quelque peu sim- pliste, car il ne tient pas compte de la culture et de la valeur de chaque immigré envers le travail, ni de bien d'autres obstacles susceptibles d'être rencontrés par eux. Si les trois raisons principales de création d'entreprise avancées par les auteures n'ont rien de surprenant, leur répartition dans la population étudiée mérite pourtant une attention : $37,4 \%$ des gens créent leur entreprise pour s'assurer d'une mobilité socio-occupationnelle, $25,1 \%$ pour rentabiliser un capital et $37,5 \%$ en vue d'enrayer les effets d'une insertion difficile et coûteuse sur le marché du travail salarié montréalais du fait d'un des désavantages dérivés de l'immigration. Cette dernière raison, ayant été retenue par environ la moitié des immigrés formés à l'étranger, confirme, dans une certaine mesure, la thèse selon laquelle les désavantages liés à l'immigration gêneraient l'entrée des immigrés sur le marché du travail des pays d'accueil. Mais, d'un autre côté, ces chiffres montrent également que la marginalité n'est plus l'unique variable explicative de l'esprit d'entreprise chez des immigrés et que devenir entrepreneur peut constituer une stratégie permettant de répondre à un désir d'autonomie dans le travail ou d'obtention de plus de revenus. La variêté des situations dans la population étudiée semble bien refléter le changement de composition sociale des flux migratoires au Canada depuis la fin des années 1970 où les politiques d'immigration favorisent davantage l'entrée d'individus scolarisés ou fortunés tout en permettant encore l'arrivée des familles des immigrés établis depuis longtemps et souvent moins éduqués.

La troisième partie du livre porte sur les modalités de création d'entreprise par des immigrés. En ce qui concerne le choix du sous-secteur d'activités, l'analyse a été effectuée en fonction de quatre variables telles que l'itinéraire occupationnel des immigrés avant la création de leur entreprise, la raison de la création, les ressources détenues à 
l'arrivée et la présence de groupe de référence dans un sous-secteur à Montréal. Ainsi, quelques faits intéressants sont à constater : près des trois quarts des immigrés étudiés choisissent un soussecteur principalement en fonction d'une expertise maintenue ou acquise au Québec; parmi les immigrés qui changent de domaine lors de la création d'entreprise, deux situations se contrastent, avec, d'un côté, ceux ayant réussi leur insertion socio-occupationnelle comme salarié au Québec et accumulé un capital, et qui choisissent librement leur nouveau secteur, et de l'autre côté, ceux qui se trouvent dans le cas de création forcée, dérivée essentiellement de désavantages. C'est surtout dans ce dernier cas que la présence d'un groupe de référence, le plus souvent dans un secteur dit "mou" (ratio capital/travail faible, concurrence forte, profitabilité faible), peut induire le choix de ce même secteur par ces immigrés. Selon les deux auteures, le désavantage lié à l'immigration porte un tiers des individus étudiés à créer leur emploi dans des sous-secteurs "mous", alors que ceux qui ne subissent pas ce désavantage le créent le plus souvent dans des domaines plus rentables et différenciés. Cependant, la révélation la plus surprenante de cette étude provient de la partie concernant la nature du réseau de mobilisation des ressources adopté par les immigrés. À ce sujet, les auteures concluent que les groupes ethniques établis à Montréal ne forment pas des marchés ségrégués de main-d'œuvre, consommation ou capital, car "aucun des individus interrogés ne s'appuie entièrement sur un réseau ethnique pour créer son emploi ou son entreprise; et le plus souvent, la main-d'ceuvre, le capital, la clientèle ou l'expertise sont trouvés en dehors de la communauté de référence ". La raison et les délais de création ainsi que la nature du métier détenu à la création sont identifiés comme les facteurs déterminants du processus menant des immigrés à choisir un type de réseau de mobilisation des ressources.
Ainsi, de cette façon, les deux auteurs ouvrent la porte à un débat important sur la capacité des immigrés à gérer des entreprises capitalistes modernes (pas " ethniques") et aussi sur leur volonté de s'intégrer aux pays d'accueil. Rappelons que le courant dominant dans la littérature actuelle sur l'entrepreneuriat immigré consiste plutôt à considérer l'appartenance ethnique comme, à la fois, le moyen incontournable de la mobilisation des ressources et le facteur principal de distribution sectorielle.

Aujourd'hui, le phénomène de la création d'entreprises par des immigrés reste encore fort peu documenté. Le livre de Helly et Ledoyen mérite que l'on s'y arrête, car il apporte de précieux renseignements à cet égard. Dans l'ensemble, ce livre est bien organisé et bien argumentê. Saluons surtout les deux auteures pour leurs efforts dans l'analyse de l'effet de situations particulières, désavantageuses, connues des immigrés et aussi pour leurs efforts dans l'examen des variables susceptibles d'expliquer la logique suivie par des immigrés dans leur création d'entreprise. Il faut néanmoins regretter un certain nombre de lacunes dans ce livre. En fait, le processus de l'immigration et de l'insertion sociooccupationnelle reprêsente, dans un sens, un grand bouleversement aussi bien pour les immigrés que pour leur pays d'accueil. Ainsi, il est difficile d'imaginer que l'on puisse réduire l'ensemble de leurs difficultés seulement à quatre désavantages êventuellement subis à leur arrivée (selon les auteures, ce sont la charge familiale, l'âge, la méconnaissance de la langue et la non-reconnaissance de l'expertise). Sans une approche plus complète, toutes analyses de corrélation risqueraient d'être biaisées. En plus, l'hypothèse de la rationalité économique des immigrés, retenue implicitement dans cette recherche semble discutable, d'autant plus que ces derniers viennent de cultures fort différentes. Notons, par ailleurs, que ce livre serait plus intéressant si le texte avait été composé de 
façon plus stimulante. Pourtant, il est clair que, même avec ces biais, ce livre comble un lacune ressentie depuis longtemps au Québec et ouvre de nombreuses voies de recherche en la matière et $\mathrm{ce}$, dans un contexte canadien où des débats restent toujours très animés sur le rôle de l'immigration et sur les contributions économiques des immigrés par rapport aux coûts engendrés pour le pays.

ZHAN SU

Université Laval

\section{Creating Economic Opportunities: The Role of Labour Standards in Industrial Restructuring}

edited by Werner SENGENBERGER and Duncan CAMPBELL, Geneva, International Institute for Labour Studies, 1994, 439 p., ISBN 92-9014-529-3 (pbk.).

While the popular and business presses seem single-minded in their portrayal of labour standards and their accompanying regulations as an obstacle to the efficient workings of the labour market, the academic community and policy-makers are divided in their perceptions and views as to whether labour standards constitute a help or hindrance to the process of industrial change and economic modernization or transformation. This debate currently suffers from at least two shortcomings. One is that the arguments for and against the economic rationality of standards are insufficiently spelled out. What is it exactly that makes standards promote and lubricate or hamper and retard adjustment? The other shortcoming is a dearth of empirical knowledge on what the actual effects of standards are for the process and economic outcome of restructuring.

To help redress this situation the International Institute for Labour Studies under its "Labour Institutions and New Industrial Organization" programme in collaboration with the European Trade Union Institute in Brussels established a working group in 1988 with the objectives of furthering conceptual work and empirical studies that would shed light on the role and value of labour standards. This volume is an attempt to lay out the case for and against labour standards in relation to economic performance.
However, one should not be under the perception that they will find a raging debate within this book since both the sponsors and contributors are advocates of using labour standards as a means of ending the vicious cycle of low wage competition. However, this is not a criticism. It was quite refreshing to read a book that seems positively iconoclastic in its views given the unrelenting onslaught against regulation of any kind, never mind an expanded role for the state.

Labour standards are viewed as a means of accommodating interests between groups of workers, firms, regions, and countries, with the key objective of preventing destructive competition in the labour market based on low or the lowering of wages and other terms of employment. In essence, the availability of undervalued labour as a result of no or inadequate labour standards is portrayed as not being conducive to productive efficiency because it provides a way of compensation for managerial, organizational, and other forms of inadequacies, delays the scraping of obsolete capital equipment and allows for price competition based on lowering wage costs. In other words, labour standards are seen as a means to bar certain possibilities of action viewed as socially damaging in order to open up and actively promote socially acceptable options for adjustment. Success in the 